ductive and epidural blockage in Emergency and Disaster Medicine. In these cases, regional anesthesia is preferable, since its use is associated with minimal negative effects on the patient's body and lower both early and late mortality, and it can be used to provide longterm analgesia.

We have created a modification of epidural anesthesia by using the development of resistance to local anesthetics. This method is based on introducing an anesthetic solution into epidural space while gradually increasing the volume and concentration of the solution used. This methods allows us to use a more effective, minimal doses of anesthetic for creation of a threshold block. Thus, it is possible to reach a sufficient quality of anesthesia with minimal hemodynamic shifts. In this case, the blockade increases slowly, with attainment a complete anesthesia by using a minimal amount of anesthetic, yet the organism has time for gradual adaptation to vegetative denervation and for the start of compensational mechanisms.

This method has been used routinely in our department, and we have been happy with the results. This type of emergency anesthesia produces perfect analgesia and reduces the depression of cardiovascular system associated with symphathetic denervation.

Key Words: conductive anaesthesia; epidural analgesia

\section{Influence of Additional Organ Failure on Outcome of Patients with Postpartum Eclamptic Coma Managed By Urgent, Isolated Ultrafiltration V.E. Bukin}

Department of Emergency Medicine, Postgraduate Medicine Institute, Zaporozhye, Ukraine

Introduction: Brain oedema caused by generalized hypoxia predetermines evolution of irreversible damage to the nervous system by eclampsia. Multiple organsystem failure syndrome is the major complication of the patients with severe eclampsia.

Our 16-year experience using Isolated Ultrafiltration (IU) in 89 postpartum patients with eclamptic coma (EC) indicated that it is a highly efficient procedure for the management of brain oedema. With the current interest in quality control and the challenge of budget restriction, it is necessary to evaluate the influence of organ failure on outcome of patients with postpartum eclamptic coma who urgently need IU.

Material and Methods: This retrospective study included 89 postpartum patients with EC (age: 17-38 years: gestation term: $27-40$ weeks). Basic intensive care measures including the administration of mechanical ventilation, magnesium, thiopental, human albumin, and drugs to lower blood pressure, were not changed during the use of IU. Isolated hemoperfusion procedures were started 6-32 hours post partum (27 pts.) or after urgent section caesarea $(62 \mathrm{pts}$.). Total IU volume: $3 \pm 0.4 \mathrm{~L}$.

We analyzed the dysfunction of organs (encephalopathy, respiratory failure, renal failure, hepatic failure, circulatory failure) on the basis of rather rough manifestations before starting therapy with IU and dependence of outcomes on the time between childbirth and beginning of IU.

Results: 20 patients had an isolated brain oedema (95\% survived), 36 patients had additional organ failures ( $91.7 \%$ survived), and 33 patients with three or more auxiliary organ system failures $(84.8 \%$ survived). Specifically, the type of organ failure influenced the chance of survival: 1) respiratory failure, $88 \%$; 2) hepatic failure, $70.5 \%$; 3) renal failure, $70.8 \%$; and 4) circulatory failure, $36.3 \%$. Of the 62 patients undergoing IU after first 6-12 hours post-partum, 59 survived, and 21 of 27 patients undergoing IU after more than 12 hours post partum survived.

Conclusion: In patients with postpartum eclamptic coma and three or more additional organ failures, the survival rate was reduced significantly. Circulatory failure influenced the outcome of these patients more than did respiratory failure and hepato-renal failure. IU application within first 6-12 hours after delivery increases survival chances in cases of eclamptic coma.

Key Words: brain oedema; isolated ultrafiltration; postpartum eclamptic coma; multiple organ failure

\section{Proposal for the Development of an Award of Excellence in Disaster Management Administered by the World Association For Disaster and Emergency Medicine (WADEM) Jennifer B. Jaumotte, MS, MPM; Ernesto A. Pretto, $M D, M P H$}

Safar Center for Resuscitation Research, Department of Anesthesiology/CCM, University of Pittsburgh, Pittsburgh, Pennsylvania USA

Recently, the world has experienced increased frequency and severity of disasters which have cost the international community hundreds of billions of dollars. Unfortunately, a substantial portion of these assets represent wasted resources due to inefficient utilization. Part of the problem is that many governmental and non governmental relief organizations lack effective management systems to coordinate, communicate, and assess their activities and outcomes.

Quality assurance methodology can improve costefficiency of response. The WADEM has established a Task Force on Quality Control of Disaster Management to develop international standards for evaluation of relief activities. ${ }^{1}$ The next step is incorporation of these standards by the leading relief organizations. To encourage the latter, we propose the establishment of an Award designed to recognize organizational performance excellence of relief actions in the following categories: A) leadership; B) information management and analysis; C) strategic planning; D) human resource development and management; E) process management; F) organizational performance assessment; and $G$ ) satisfaction of relief recipients, donors, and other beneficiaries.

The premise of an International Quality Award is parallel to the Malcolm Baldridge National Quality of the USA Commerce Department that recognizes organizations for performance excellence. Organizations 
interested in participating in the WADEM disaster management award process would be asked to submit an application that includes a self-evaluation package based on quality control criteria. In responding to these, each applicant would be expected to provide information on improvement processes and results. Information submitted would be kept confidential. Each organization would need to demonstrate that its approaches are effective and can be replicated or adopted by others. The criteria are designed not only to serve as a reliable basis for making awards, but also to permit an assessment of the organization's overall performance management system. All applicants would receive an appraisal of their organization's quality programs with recommendations for improvement where needed. A site visit of the best organizations (finalists) as decided by a panel of evaluators would select the winner.

1. Prehospital and Disaster Medicine 1996;11(2):16-24.

Key Words: award; disaster management; disaster relief; disaster response; quality assurance; quality control

\section{Syringe Or Bulb?}

Three Oesophageal Detector Devices

W.H. Maleck; ${ }^{1}$ K.P. Koetter; ${ }^{2}$ A. Jatzko; ${ }^{1}$

G.A. Petroianu ${ }^{1}$

1. Pharmacology, Klinikum, Mannheim, Germany 2. Juliusspital, Würzburg, Germany

Introduction: The Oesophageal Detector Device (ODD) is a suction device (syringe or bulb) to be fitted to the endotracheal tube. Air is aspirated easily from the rigid trachea, but not from the collapsing oesophagus. Undetected oesophageal tubes are very rare $(<1 \%)$ with both types of ODDs. Unconfirmed tracheal tubes occur in about $1 \%$ with syringe ODDs, but are more common with bulb ODDs. Proponents of the syringe argue using these lower failure rates. Proponents of the bulb argue using its simplicity and speed of use. No comparison of decision times for syringe vs. bulb has been published.

Methods: We compared a bulb-type ODD, the Ambu TubeCheck-Bulb (TCB) with two syringe-type ODDs, the Ambu TubeCheck-Syringe (TCS) and a self-made syringe type ODD (SMS). Fourteen EMT-Paramedics and 14 nurses, blinded to tube position, used each device once on a Ambu Intubation Trainer with a TubeCheck Training Kit. Time was recorded from the initial touch of the device to the decision of tube position. Each participant scored the handling of each device on a 6-point scale routinely used for school marks in Germany $(1=$ very good, $6=$ insufficient). The decision-time of the other two devices was compared to that for the TCB using the rank order test for paired observations. Handling of the other two devices was compared to TCB using the sign test for paired observations. A pvalue of $<0.05$ after Bonferroni-correction $\left({ }^{*} 4\right)$ was considered statistically significant.

Results: All decisions were correct. Mean \pm one SD decision-times were: TCB, $4.6 \pm 1.7$ seconds; TCS, 4.7 \pm 1.6 seconds; and SMS $5.1 \pm 1.3$ seconds. The time difference between TCB and SMS was significant statisti- cally $(p<0.02)$. Mean \pm SD handling scores were: TCB, $1.7 \pm 1.0$; TCS, $2.4 \pm 1.0$; and for SMS, $2.7 \pm 1.0$. The handling difference between TCB and SMS was statistically significant $(p<0.01)$.

Conclusion: The use of the TCB was quicker and easier was than was use of the SMS. There was a trend to quicker and easier use of the TCB compared with the TCS. From a clinical point of view, however, differences in mean decision times of less than 1 second seem irrelevant. The same applies to the handling scores as the mean value for the scores for the SMS of 2.7 equals "satisfactory".

Key Words: ergonomics; esophageal detector device; oesophageal detector device; out-of-hospital; tracheal intubation

\section{Poisoning with Cholinesterase Inhibitors- A Possible Cause for a Disaster \\ Detlef Nick; Peter Enders \\ Bundeswehrzentralkrankenhaus, Koblenz, Germany}

Poisoning with an insecticide still is one of the most often reported poisonings. Clinically, the most relevant are poisonings with cholinesterase-inhibitors $(\mathrm{ChE}-\mathrm{I})$, especially the carbamates and organic phosphates. Poisoning with $\mathrm{ChE}-\mathrm{I}$ manifests by maximum stimulation of the vagus nerve with corresponding clinical symptoms, muscular symptoms, and irritation of the CNS.

The possibility that the environment of the affected person is contaminated as well as that of the EMS personnel, and of the primary unconcerned persons is an important consideration for handling of ChE-I poisoning by the emergency medical services. Depending on the type, ChE-I can be absorbed very easily through the intact skin or when inhaled. They can cause symptoms of poisoning even in low doses.

ChE-I are readily available as insecticides. If the acute-toxic potential is taken into consideration, such poisonings by suicide or accident can be expected; not rarely, especially in regions with a high consumption of insecticides such as the wine-growing countries along Rhine and Mosell.

Poisoning with ChE-I is to be considered always as an ongoing event. Even if only suspicious about a poisoning with ChE-I, clear steps which focus on avoiding the progression of the damage have to be taken by the EMS personnel first arriving on the scene. These steps to be taken are: 1) self-protection by wearing an antichemicals overall (if available) respectively suitable clothing, gloves, galoshes, and rubber cover; 2) airwayprotection using common anti-ABC-masks (available e.g., at the German Police or the German Federal Armed Forces), which are effective against all customary insecticides with the normally used gas-filters; 3 ) wherever possible, transport of the persons afflicted into the open air; 4) removal of person not involved; 5) if needed, the fire department should be consulted (e.g., rescue from contaminated rooms); 6) external decontamination of the poisoned persons (soap, warm water), removal of contaminated clothing; 7) should the occasion arise, prophylaxis of toxic lung-edema, even if only suspected. 